\title{
NOTES
}

\section{Characterization of Ethynylene-Containing Polyamide Using Size Exclusion Chromatography with Light Scattering Detector}

\author{
Shinji Watanabe, Masaru Tanabe, Mitsutoshi JikeI, \\ Masa-aki KaKimoto, and Yoshio IMAI* \\ Department of Organic and Polymeric Materials, Tokyo Institute of Technology, \\ Meguro-ku, Tokyo 152, Japan
}

(Received February 17, 1995)

\begin{abstract}
KEY WORDS Size Exclusion Chromatography / Light Scattering / Ethynylene Containing Polymer / Branched Polymer /
\end{abstract}

Size exclusion chromatography (SEC) is one of the most frequently used and most effective techniques for the determination of molecular weights and molecular weight distributions of polymers. However, it is difficult to derive absolute molecular weights by using conventional detectors (ultraviolet (UV) or refractive index (RI)), because of not only band and peak broadening of the SEC chromatogram, but also difference of the structures between a polymer sample and a calibration polymer. To address these problems, a laser low angle light scattering detector was started to use for a SEC two decades ago. ${ }^{1}$ It can yield an absolute molecular weight, molecular weight distribution without necessity of calibration of a standard polymer, and detect a high molecular weight component which cannot be detected with RI or UV detector. ${ }^{2-4}$ Recently Wyatt introduced a multiangle light scattering detector into a SEC apparatus. It allows measurements of scattered light intensity at various angles at once and can give conformational data such as a root mean square radius and branching ratio of the polymer. ${ }^{5-7}$

Recently much attention is being paid for the synthesis of ethynylene containing poly- mers by the palladium-catalyzed cross-coupling reaction. These polymers are useful for conducting polymers, ${ }^{8,9}$ third-order nonlinear optical materials, ${ }^{9}$ and thermo-plastics. ${ }^{10}$ Particularly, they are promising materials for thermo-setting high temperature plastics because they have not only rigid main chain but also thermo-curable internal ethynylene unit. We have already reported the synthesis of ethynylene-containing polyamides by using palladium catalyst, which show good thermal property and thermal curing reaction. ${ }^{11}$ However, their molecular weight distributions determined by a SEC with a RI detector were reported to be extremely large. This may be due to the cross-linking reaction via the internal ethynylene groups during the polymerization though it can not be confirmed by either NMR or IR. The purpose of this study is to measure molecular weights of these polymers and to discuss the polymer structure (branched structure, microgels, and so on) by employing a SEC equipped with a multiangle light scattering (LS) photometer. The polymer structures formed under various polymerization conditions are also discussed.

* To whom correspondence should be addressed. 


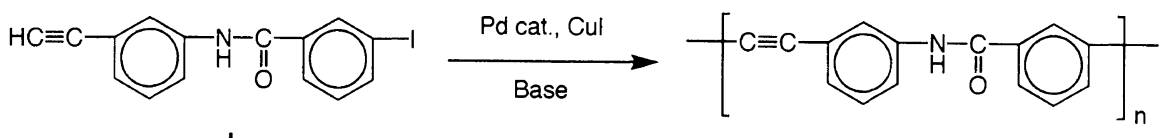

I

Scheme 1.

\section{EXPERIMENTAL}

\section{Polymerization}

The polymer was synthesized following the previous paper (Scheme 1). ${ }^{8}$ Typically, a mixture of $0.694 \mathrm{~g}(2.00 \mathrm{mmol})$ of $N-\left(3^{\prime}-\right.$ ethynylphenyl)-3-iodobenzamide I, $14 \mathrm{mg}$ $(0.020 \mathrm{mmol})$ of bis(triphenylphosphine) palladium(II) dichloride, $15 \mathrm{mg}(0.079 \mathrm{mmol})$ of cuprous iodide, $26 \mathrm{mg}(0.099 \mathrm{mmol})$ of triphenylphosphine, $0.55 \mathrm{ml}(3.97 \mathrm{mmol})$ of triethylamine, and $10 \mathrm{ml}$ of $N, N$-dimethylacetamide (DMAc) was stirred at $100^{\circ} \mathrm{C}$ for $4 \mathrm{~h}$ under nitrogen atmosphere. The polymer solution was poured into $300 \mathrm{ml}$ of methanol and the polymer was washed with refluxing methanol. The yellow powdery polymer $(0.41 \mathrm{~g}$ : $94 \%$ ) was obtained. The inherent viscosity of the polymer was $0.43 \mathrm{dlg}^{-1}$ in DMAc, measured at a concentration of $0.5 \mathrm{~g} \mathrm{dl}^{-1}$ at $30^{\circ} \mathrm{C}$.

\section{SEC Measurement}

SEC measurements were performed by using a JASCO HPLC BIP-I apparatus equipped with a mini DAWN light scattering photometer (Wyatt Technology Co.) containing a fixed array of three angle detectors $\left(45,90\right.$, and $\left.135^{\circ}\right)$, a Shimadzu RID-6A refractive index detector, and polystyrene-divinylbenzene columns (two Shodex KD-806 and KD-802). The specific refractive index increment $(\mathrm{d} n / \mathrm{d} c)$ of the polymer in $N, N$-dimethylformamide (DMF) was determined by a Wyatt/Optilab 903 (Wyatt Technology Co.) and was $0.319 \mathrm{ml} \mathrm{g}^{-1}$. The polymer for the SEC measurement was dissolved in DMF containing $0.01 \mathrm{moll}^{-1}$ of lithium bromide at a concentration of $0.2-$ $0.4 \mathrm{mg} \mathrm{ml}^{-1}$, and then the solution was sonicated for $30 \mathrm{~min}$ and was filtered with a Millipore filter. The mobile phase was DMF

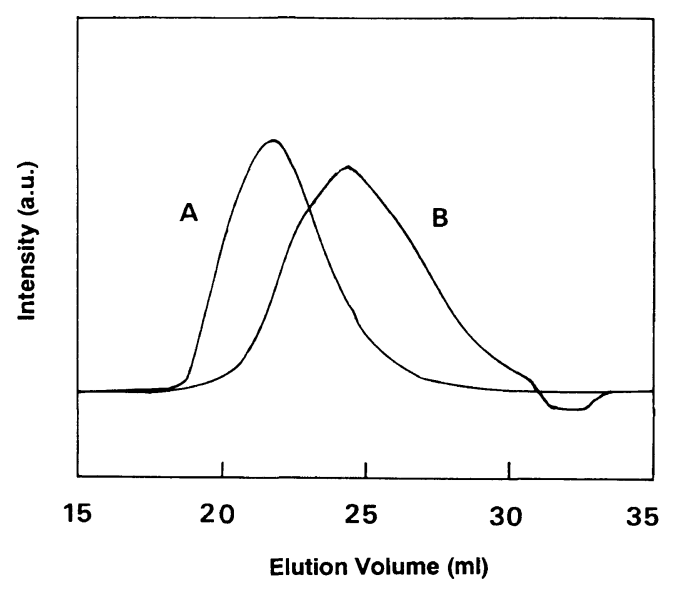

Figure 1. Size exclusion chromatograms of polyamide (Run No. 1) obtained by (A) light scattering at $90^{\circ}$ and (B) refractive index detectors.

containing $0.01 \mathrm{moll}^{-1}$ of lithium bromide with a flow rate of $1.0 \mathrm{ml} \mathrm{min} \mathrm{m}^{-1}$ and about $2 \mathrm{ml}$ of the polymer solution was injected into columns.

\section{RESULTS AND DISCUSSION}

Figure 1 shows the profiles of SEC curves monitored by both light scattering at $90^{\circ}$ and refractive index detectors. The light scattering photometer was sensitive to high molecular weight fraction compared with refractive index detector, because the intensity of light scattering signal is proportional to both the molecular weight and the concentration of the polymer. Table I summarizes weight average molecular weights $\left(M_{w}\right)$ and molecular weight distributions $\left(M_{w} / M_{n}\right)$ of the polymers obtained under various conditions such as catalysts, bases, and solvents, determined by a SEC measurement with a light scattering photometer and an RI detector based on 
Table I. Molecular weights of polymers

\begin{tabular}{|c|c|c|c|c|c|c|c|c|}
\hline Run No. & Catalyst & Base & Solvent & $\eta_{\text {inh }} / \mathrm{dl} \mathrm{g}^{-1 \mathrm{a}}$ & $M_{w}{ }^{\mathrm{b}}$ & $M_{w} / M_{n}^{\mathrm{b}}$ & $M_{w}{ }^{\mathrm{c}}$ & $M_{w} / M_{n}{ }^{\mathrm{c}}$ \\
\hline 1 & $\mathrm{PdCl}_{2}\left(\mathrm{PPh}_{3}\right)_{2}$ & Triethylamine & DMAc & 0.43 & 125000 & 4.42 & 167000 & 5.31 \\
\hline 2 & $\mathrm{Pd}(\mathrm{OAc})_{2}$ & Triethylamine & DMAc & 0.19 & 72600 & 1.97 & 126000 & 5.88 \\
\hline 3 & $\mathrm{Pd}\left(\mathrm{PPh}_{3}\right)_{4}$ & Triethylamine & DMAc & 0.18 & 45500 & 1.32 & - & - \\
\hline 4 & $\mathrm{Pd}\left(\mathrm{PPh}_{3}\right)_{4}$ & Tri- $n$-propylamine & DMAc & 0.19 & 437000 & 6.93 & 244000 & 11.45 \\
\hline 5 & $\mathrm{PdCl}_{2}\left(\mathrm{PPh}_{3}\right)_{2}$ & BDMAN $^{d}$ & DMAc & 0.08 & 11700 & 2.26 & - & - \\
\hline 6 & $\mathrm{PdCl}_{2}\left(\mathrm{PPh}_{3}\right)_{2}$ & Triethylamine & DMF & 0.29 & 355000 & 3.94 & 151000 & 5.47 \\
\hline 7 & $\mathrm{PdCl}_{2}\left(\mathrm{PPh}_{3}\right)_{2}$ & Triethylamine & NMP & 0.24 & 47300 & 1.98 & - & - \\
\hline
\end{tabular}

${ }^{\mathrm{a}}$ Inherent viscosity measured at a concentration of $0.5 \mathrm{~g} \mathrm{dl}^{-1}$ in DMAc at $30^{\circ} \mathrm{C}$. ${ }^{\mathrm{b}}$ Measured with SEC-LS eluted by DMF. ' Measured with SEC eluted by DMF based on polystyrene standard. ${ }^{\mathrm{d}}$ 1,8-Bis(dimethylamino)naphthalene.

polystyrene standards eluted with DMF. The molecular weight distributions of the polymers based on polystyrene standard were wider than those estimated by using the light scattering photometer, and especially the deviations in Runs No. 2 and 4 are large. A few reasons may cause such a large deviation. First, SEC-LS can derive the molecular weight distribution without the band broadening factor for chromatograms. ${ }^{1,2}$ And if the column resolution is not perfect, the molecular weight distribution determined by a SEC-LS will be smaller than the true one. ${ }^{1,2}$ The other reason is a structural difference between polymer II and polystyrene standard. Because polymer II has a very rigid main chain, its implication between molecular weight and elution volume must be different from that for polystyrene standards.

We also measured inherent viscosity of the polymers, which did not correlate with the weight average molecular weights determined with a SEC-LS (Runs No. 4 and 6). Since this type of polymer ready crosslinked by the thermal treatment at $270^{\circ} \mathrm{C}$ for $7 \mathrm{~h},{ }^{8}$ some crosslinking reaction might occur during the polycondensation. Plots of log root mean square radius $\left(\left\langle r_{\mathrm{g}}{ }^{2}\right\rangle^{1 / 2}\right)$ as a function of $\log$ molecular weight $(M)$ are shown in Figure 2. For a sufficiently high-molecular-weight linear polymer, a plot of $\log \left\langle r_{\mathrm{g}}{ }^{2}\right\rangle^{1 / 2}$ against $\log M$ should make a straight line and the slope of the line should indicate the polymer conformation. The small slope implies that crosslinking

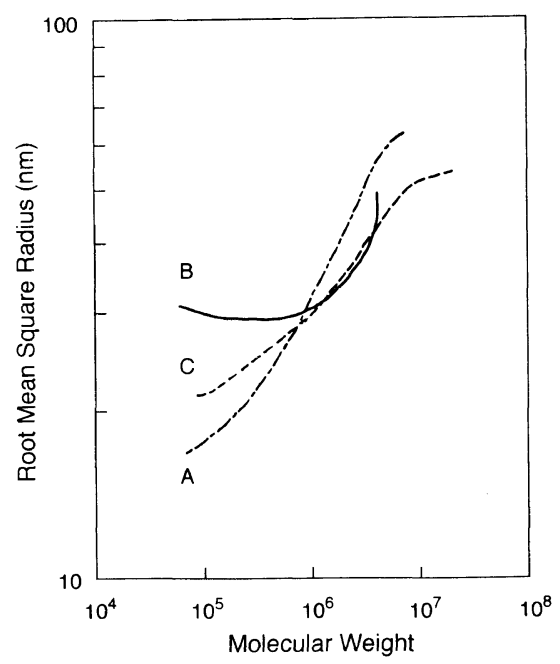

Figure 2. Root mean square radius versus molecular weight of polymers (A, Run No. 1; B, Run No. 4; C, Run No. 6).

reactions occur during the polymerization, because the molecular weight increases without so much change of the molecular size. The polymer obtained in DMAc (Run No. 1) and the polymer in DMF (Run No. 6) showed almost straight lines; however their slopes were very different. The slop of Run No. 6 was much smaller than that of Run No. 1. This implies that the polymer obtained in DMF contains some branched structures. The curve of the polymer using tri- $n$-propylamine (Run No. 4) was apart from a straight line. The result shows this polymer should include branched fractions, microgels, or networks. ${ }^{6}$ The poly- 
condensation using tri- $n$-propylamine or DMF (Run No. 4 or 6) yielded the polymer having low inherent viscosity value compared with the molecular weight, which could account for the formation of some branched fractions or coagulation forms in solution as estimated by the SEC-LS method. Such a structural information could not be obtained by NMR or IR, but by SEC-LS.

In conclusion, a SEC-LS can yield a true molecular weight and molecular weight distribution of a polymer, which is very important for unknown polymers especially, if the structure and the solubility of the polymer are different from those of the standard polymer. In this case, there are large deviations between the molecular weight distributions determined by polystyrene standards and those by the light scattering detector. A SEC-LS is also a powerful method to estimate some side reactions during the polymerization which may especially contain any crosslinking reactions.

\section{REFERENCES}

1. A. C. Ouano and W. Kaye, J. Polym. Sci., Polym. Chem. Ed., 12, 1151 (1974).

2. A. C. Ouano, J. Chromatogr., 118, 303 (1976).

3. P. M. Cotts and R. Siemens, Polymer, 32, 3052 (1991).

4. A. C. Ouano, J. Colloid Interface Sci., 63, 275 (1978).

5. S. H. Kim and P. M. Cotts, J. Appl. Polym. Sci., 42, 217 (1991).

6. P. J. Wyatt, Analytica Chim. Acta, 272, 1 (1993); P. J. Wyatt, Special Publications Royal Soc. Chem., 99, 35 (1992).

7. T. Kato, A. Kanda, A. Takahashi, I. Noda, S. Maki, and M. Nagasawa, Polym. J., 11, 575 (1979).

8. M. Takagi, K. Kizu, Y. Miyazaki, T. Maruyama, K. Kubota, and T. Yamamoto, Chem. Lett., 913 (1993).

9. T. Yamamoto, W. Yamada, M. Takagi, K. Kizu, T. Maruyama, N. Ooba, S. Tomaru, T. Kurihara, T. Kaino, and K. Kubota, Macromolecules, 27, 6620 (1994).

10. S. J. Havens and P. M. Hergenrother, J. Polym. Sci., Polym. Lett., 23, 587 (1985).

11. S. Watanabe, M. Tanabe, M. Kakimoto, and Y. Imai, J. Polym. Sci., A, Polym. Chem., 32, 987 (1994). 